\title{
Revisiting the $\bar{t} h h$ channel at the FCC-hh
}

\author{
Shankha Banerjee ${ }^{*}{ }^{*}$ Frank Krauss, ${ }^{\dagger}$ and Michael Spannowsky ${ }^{\ddagger}$ \\ Institute of Particle Physics Phenomenology, Durham University, Durham DH1 3LE, United Kingdom
}

(Received 23 April 2019; published 22 October 2019)

\begin{abstract}
The exploration of the scalar sector of the Standard Model is at the core of current and future science programs at collider experiments, with increasing focus on the self-interaction of the Higgs boson. This important parameter of the Higgs sector can be measured in various channels, among the production of a Higgs boson associated with a top-quark pair, $\bar{t} t h h$. In this paper we study this channel and its potential to measure or constrain the self-coupling and possible new physics contributions at a future $100 \mathrm{TeV}$ protonproton collider. Analyzing this highly complex final state adds to the sensitivity for enhanced self-coupling interactions, and we argue that a measurement of this process is a necessity to constrain blind directions in the multidimensional parameter space of well-motivated new physics scenarios.
\end{abstract}

DOI: 10.1103/PhysRevD.100.073012

\section{INTRODUCTION}

After the discovery of the Higgs boson [1,2] with a mass of $m_{h} \simeq 125 \mathrm{GeV}$, the focus of contemporary and future particle phenomenology shifted to the determination of its properties. Early on, spin and $C P$ properties have already been found to be in extremely good agreement with Standard Model (SM) expectations [3-6], and the couplings of the Higgs boson to other particles, in particular the heavy gauge bosons and the third-generation fermions, are increasingly precisely measured [7-9], thereby reducing the parameter space for extensions of the Standard Model [10-13]. This leaves the form and parameters of the Higgs potential, and in particular the Higgs self-coupling, as the experimentally least constrained sector of the Standard Model. It is therefore not surprising that measurements of or constraints to the triple-Higgs coupling are one of the center pieces of ongoing efforts for the high-luminosity run of the LHC and an important part of particle phenomenology at possible future collider experiments. If the Higgs self-coupling is the only modification to the Standard Model, various ways have been proposed at existing and future colliders to search for this interaction. These approaches can be classified into three categories: in processes without Higgs bosons in the final state, electroweak precision observables can set a limit to $\lambda_{h h h}$ [14-16];

\footnotetext{
*shankha.banerjee@durham.ac.uk

frank.krauss@durham.ac.uk

\#michael.spannowsky@durham.ac.uk
}

Published by the American Physical Society under the terms of the Creative Commons Attribution 4.0 International license. Further distribution of this work must maintain attribution to the author(s) and the published article's title, journal citation, and DOI. Funded by SCOAP ${ }^{3}$. higher-order corrections in single-Higgs production processes [17-19] constrain the Higgs self-coupling; and double-Higgs production processes will provide direct sensitivity on this coupling in upcoming LHC and possible future high-energy collider runs [20-27], while the latter are expected to provide the best sensitivity on $\lambda_{h h h}$ during the LHC's high-luminosity runs.

Within the class of multi-Higgs production processes, the overwhelming focus to date was directed toward the channel with the largest cross section, i.e., Higgs-boson pair production in gluon fusion, while other channels, such as Higgs-pair production in association with other particles, e.g., $p p \rightarrow h h j j$ [28-30] or $p p \rightarrow t \bar{t} h h$ [31,32], have been somewhat neglected. In Refs. [31,32] it has been found that the $t \bar{t} h h$ channel at the $14 \mathrm{TeV}$ high-luminosity run of the LHC may provide welcome additional statistical power for a determination of the trilinear Higgs coupling. The feature that sets this channel apart from the gluon-induced Higgspair production process or the weak-boson induced production of $h h j j$ arises due to the absence of a reduced cross section for large values of $\lambda_{h h h}$ [33]. Thus, $t \bar{t} h h$ could be particularly useful in setting a stringent limit to enhanced self-interactions of the Higgs boson.

A further motivation to measure $t \bar{t} h h$ final states arises when modifications of Higgs interactions originate in models where the Higgs field is realized in a nonlinear way, e.g., composite Higgs models [34-36]. There, the $t \bar{t} h$ and $t \bar{t} h h$ couplings are decorrelated [37,38], leading to a blind direction in the parameter space of effective operators when only probing them through the top-associated single Higgs production process, $p p \rightarrow t t h$. Thus, to rule out such a scenario conclusively, measuring the $t \bar{t} h h$ process during future LHC runs or at future colliders is not optional but a necessity. 


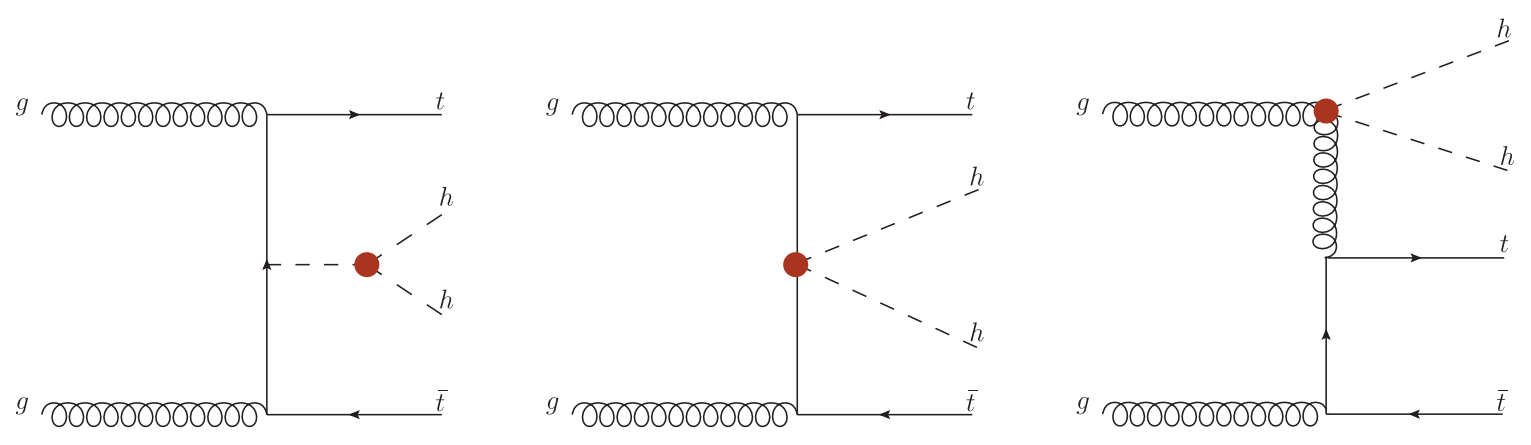

FIG. 1. Feynman diagrams [53] showing the impact of the three effective vertices, viz. $h h h, t \bar{t} h h$, and $g g h h$.

In the present work we revisit the proposal of [31] by extending it to the potential future FCC-hh $100 \mathrm{TeV} p p$ collider and including the study of contributions from effective $t \bar{t} h h$ interactions. We will focus on the scenario where both Higgs bosons decay into bottom quarks while one of the top quarks decays leptonically and the other hadronically. ${ }^{1}$ Owing to the increase in energy, we will see that this channel is competitive with various other di-Higgs channels $[24,26,39-46]$ in constraining the trilinear Higgs self-coupling at the $100 \mathrm{TeV}$ collider. This increase in cross section due to energy is, of course, also a feature of the backgrounds, and we therefore substantially increase their discussion.

Using the formalism of effective field theories, with a strongly coupled UV completion in mind, in Sec. II we describe why obtaining a direct measurement of the $t \bar{t} h h$ final state at current or future colliders is of importance to obtain meaningful constraints in the top-Higgs sector. In Sec. III we describe the technical framework used. The analysis steps, reconstruction efficiencies, and kinematic features of the signal and the background are detailed in Sec. IV. Finally, we offer our conclusions in Sec. V.

\section{EFFECTIVE FIELD THEORY FORMALISM}

Extensions of the Standard Model can lead to various modifications of Higgs interactions. Some of the most popular are composite Higgs models, which assume that the Higgs boson is a pseudo-Nambu-Goldstone boson of a strongly coupled UV completion. The most general effective field theory that describes the low-energy effects of a strongly coupled embedding of the Standard Model is the electroweak chiral Lagrangian (ew $\chi \mathrm{L})$ [47-51]. Here, the $S U(2) \times U(1)$ symmetry is nonlinearly realized,

\footnotetext{
${ }^{1}$ A fully leptonic $t \bar{t}$ even though much cleaner suffers from a reduction in the total rate. A fully hadronic scenario, on the other hand, will entail large QCD backgrounds. However, the total sensitivity will increase if we study the leptonic, semileptonic, and hadronic scenarios in conjunction. This we leave for a more comprehensive future study.
}

TABLE I. Relationship between the $h h h, t \bar{t} h h$, and $g g h h$ vertices in three different bases $[38,52,54]$, where $\xi \equiv(v / f)^{2}$.

\begin{tabular}{|c|c|c|c|}
\hline Coupling & $\begin{array}{c}\text { Nonlinear } \\
\text { EFT }\end{array}$ & $\begin{array}{l}\text { Simplified } \\
\text { Lagrangian }\end{array}$ & SILH \\
\hline$h h h$ & $d_{3}$ & $\kappa_{\lambda}$ & $1+\left(c_{6}-c_{\tau} / 4-3 c_{H} / 2\right) \xi$ \\
\hline$t \bar{t} h h$ & $c_{2}$ & $-\frac{\sqrt{2} v}{y_{t}} \kappa_{t \bar{t} h h}$ & $-\left(c_{H}+3 c_{y}+c_{\tau} / 4\right) \xi / 2$ \\
\hline$g g h h$ & $k_{2 g}$ & $-\frac{12 \pi^{2} v^{2}}{g_{s}^{2}} \kappa_{g g h h}$ & $3 c_{g}\left(\frac{y_{t}^{2}}{g_{\rho}^{2}}\right) \xi$ \\
\hline
\end{tabular}

$$
\Sigma(x)=e^{i \sigma^{a} \phi^{a}(x) / v},
$$

with the Goldstone bosons $\phi^{a}(a=1,2,3)$ and the Pauli matrices $\sigma^{a}$. After introducing a scalar field that transforms linearly under the custodial symmetry, the Lagrangian contains $^{2}$

$$
\begin{aligned}
\mathcal{L}^{\mathrm{ew} \chi} \supset & -V(h)+\frac{g_{s}^{2}}{48 \pi^{2}} G_{\mu \nu}^{a} G_{a}^{\mu \nu}\left(k_{g} \frac{h}{v}+\frac{1}{2} k_{2 g} \frac{h^{2}}{v^{2}}+\cdots\right) \\
& -\frac{v}{\sqrt{2}}\left(\bar{u}_{L}^{i} \bar{d}_{L}^{i}\right) \Sigma\left[1+c \frac{h}{v}+c_{2} \frac{h^{2}}{v^{2}}+\cdots\right] \\
& \times\left(\begin{array}{c}
y_{i j}^{u} u_{R}^{j} \\
y_{i j}^{d} d_{R}^{j}
\end{array}\right)+\text { H.c. }
\end{aligned}
$$

with

$$
V(h)=\frac{1}{2} m_{h}^{2} h^{2}+d_{3} \frac{m_{h}^{2}}{2 v} h^{3}+d_{4} \frac{m_{h}^{2}}{8 v^{2}} h^{4}+\cdots .
$$

Focusing on contributions of effective operators to the top-Higgs sector we find five operators to be of imminent importance, i.e., the ones associated with the coefficients $k_{g}, k_{2 g}, c, c_{2}$, and $d_{3}$. While $k_{g}$ and $c$ can be constrained in various single-Higgs production processes, e.g., gluon

\footnotetext{
${ }^{2}$ We follow the notation of [38]. In [38] it is also shown how the coefficients $k_{g}, k_{2 g}, c, c_{2}$, and $d_{3}$ translate to the effective dimension-six operators of a linearized sigma model, the socalled strongly-interacting light Higgs (SILH) parametrization [52].
} 

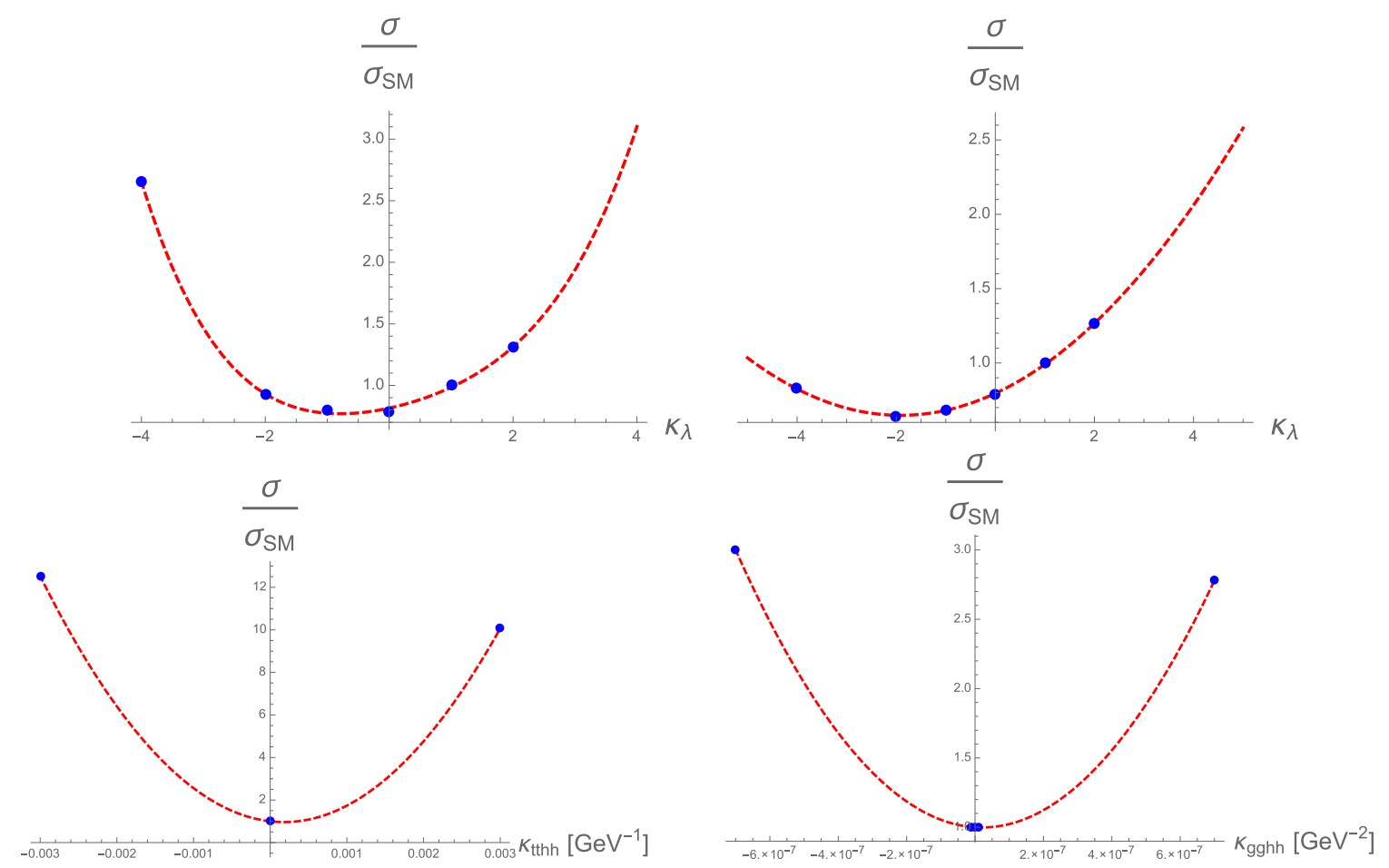

FIG. 2. $\sigma / \sigma_{\mathrm{SM}}$ as a function of $\kappa_{\lambda}$ (top left at $100 \mathrm{TeV}$ and top right at $14 \mathrm{TeV}$ ), $\kappa_{\text {tthh }}\left[\mathrm{GeV}^{-1}\right]$ (bottom left at $100 \mathrm{TeV}$ ), and $\kappa_{\text {gghh }}$ $\left[\mathrm{GeV}^{-2}\right]$ (bottom right at $100 \mathrm{TeV}$ ). For the $100 \mathrm{TeV}$ case, $\sigma_{\mathrm{SM}}=16.4 \mathrm{fb}$ (this includes $h \rightarrow b \bar{b}$ for both Higgs bosons). The corresponding cross section for the $14 \mathrm{TeV}$ scenario is $0.22 \mathrm{fb}$.

fusion, vector-boson fusion, or top-associated singleHiggs production, the coefficients $k_{2 g}, c_{2}$, and $d_{3}$ rely at leading-order predominantly on double-Higgs production processes to be tensioned with data. Thus, to overconstrain the parameter space of $\mathcal{L}^{\mathrm{ew}} \chi$ it is necessary to access as many double-Higgs channels as possible, i.e., $p p \rightarrow$ $h h, p p \rightarrow h h j j$, and $p p \rightarrow t \bar{t} h h$. The process $p p \rightarrow t \bar{t} h h$ is of particular relevance to constrain $c_{2}$, as it is the only process of appreciable cross section where this coefficient can be constrained at tree level. However, it is to be noted that the one-loop gluon fusion production of di-Higgs (at LO) also affects $c_{2}$, albeit with a different weight from $t \bar{t} h h$. The Feynman diagrams showing the modified vertices are shown in Fig. 1.

In this paper, we work with the simplified Lagrangian

$$
\begin{aligned}
\mathcal{L}^{\text {simp }}= & \mathcal{L}^{\mathrm{SM}}+\left(1-\kappa_{\lambda}\right) \lambda_{\mathrm{SM}} h^{3}+\kappa_{t \bar{t} h h}\left(\bar{t}_{L} t_{R} h^{2}+\text { H.c. }\right) \\
& -\frac{1}{8} \kappa_{g g h h} G_{\mu \nu}^{\mathrm{a}} G_{\mathrm{a}}^{\mu \nu} h^{2},
\end{aligned}
$$

where $\lambda_{\mathrm{SM}}=\lambda v=\frac{m_{h}^{2} 3}{2 v}$ and $\kappa_{\lambda}=\lambda_{\mathrm{BSM}} / \lambda_{\mathrm{SM}}$. In Table I, we show the relations between the various bases. For reference, we also include the relationship with the SILH basis $[38,52,54,55]$, which corresponds to a linearized sigma model. The SILH Lagrangian is defined as [38]

\footnotetext{
${ }^{3}$ The Higgs potential in the SM can be written as $V_{H}=$ $\frac{1}{2} 2 \lambda v^{2} h^{2}+\lambda v h^{3}+\frac{\lambda}{4} h^{4}$.
}

$$
\begin{aligned}
\mathcal{L}_{\mathrm{SILH}}= & \frac{c_{H}}{2 f^{2}} \partial^{\mu}\left(H^{\dagger} H\right) \partial_{\mu}\left(H^{\dagger} H\right)+\frac{c_{\tau}}{2 f^{2}} H^{\dagger} H\left(D^{\mu} H\right)^{\dagger}\left(D_{\mu} H\right) \\
& -\frac{c_{6} \lambda}{f^{2}}\left(H^{\dagger} H\right)^{3}+\left(\frac{c_{y} y_{f}}{f^{2}} H^{\dagger} H \bar{f}_{L} H f_{R}+\text { H.c. }\right) \\
& +\frac{c_{g} g_{s}^{2}}{16 \pi^{2} f^{2}} \frac{y_{t}^{2}}{g_{\rho}^{2}} H^{\dagger} H G_{\mu \nu}^{a} G^{a \mu \nu}+\frac{c_{\gamma} g^{2}}{16 \pi^{2} f^{2}} \frac{g^{2}}{g_{\rho}^{2}} H^{\dagger} H B_{\mu \nu} B^{\mu \nu},
\end{aligned}
$$

where $g, g_{s}$, and $g^{\prime}$ are, respectively, the $S U(2)_{L}, S U(3)_{c}$, and $U(1)_{Y}$ couplings in the SM, $g_{\rho}$ is the coupling of the strongly interacting sector, and $\lambda$ and $y_{f}$ are, respectively, the Higgs quartic coupling and the Yukawa coupling.

We show the ratio of signal cross sections with respect to the SM expectation as a function of $\kappa_{\lambda}, \kappa_{t \bar{t} h h}$, and $\kappa_{g g h h}$ in Fig. 2. One can see that the cross section increases with $\lambda>\lambda_{\mathrm{SM}}$. We validated our setup by checking this ratio $\left(\sigma / \sigma_{\mathrm{SM}}\right)$ at $14 \mathrm{TeV}$ with Ref. [33]. It is interesting to note that the nature of the growth of the cross section for negative values of $\lambda$ changes significantly upon going from a $14 \mathrm{TeV}$ machine to a $100 \mathrm{TeV}$ machine. In the SILH Lagrangian, the coupling modifying the ggh coupling also contributes to the $g g h$ coupling. Allowing for a $10 \%$ modification in the $14 \mathrm{TeV} g g \rightarrow h$ cross section, we find that $\kappa_{g g h h}$ is very strongly constrained. Thus, in the analysis, we only consider the couplings modifying the trilinear Higgs coupling and the $t \bar{t} h h$ vertex. 
Before moving on with the analysis, we dedicate this paragraph to explain the differences between the linear and the nonlinear realizations of the effective field theory (EFT). Upon considering a linear EFT, the Higgs is essentially considered to be the part of a doublet (as in SM) and the couplings affecting the $g g h$ and the $g g h h$ vertices are coming from the same set of Wilson coefficients. This is the same for the $t \bar{t} h$ and the $t \bar{t} h h$ couplings and also for the trilinear and the quartic Higgs couplings. Hence, in a linear realization of the EFT, we have a fewer number of parameters to constrain. ${ }^{4}$ In contrast, when one considers a nonlinear realization of the EFT, as we will be considering for most of this work, all these couplings are independent and a priori there are no preferential directions. In Ref. [41], the $p p \rightarrow$ $h h$ process has been studied in the nonlinear EFT scenario where the various parameters have been constrained upon studying the $h h \rightarrow b \bar{b} \gamma \gamma$ channel. From Ref. [41], it can be seen that $\kappa_{\text {gghh }}$ is constrained to around $\mathcal{O}\left(10^{-8}\right) \mathrm{GeV}^{-2}$ from the $p p \rightarrow h h$ production, which is an order of magnitude stronger than our assumption. ${ }^{5}$ It is important to realize that various double Higgs processes give us constraints on different linear combinations of these couplings and one encounters blind directions. This necessitates the study of all double Higgs processes in order to break these blind directions and to combine the results to obtain stronger constraints. In this first work for the $p p \rightarrow t \bar{t} h h$ channel, we consider the nonlinear realization of the EFT and treat the couplings independent of each other.

\section{THE MONTE CARLO SETUP}

The final state in our analysis results from the decays of the two top quarks and the two Higgs bosons into six $b$-tagged jets, one isolated lepton, missing transverse momentum, and at least two extra jets that are not $b$ tagged. This leaves a wide range of backgrounds to be considered; see below. In all channels, potential additional jets may give rise to required final state particles, either by jet radiation mimicking the light jets stemming from the hadronically decaying top quark or by gluons splitting into $b$-quark pairs, yielding $b$-tagged jets. In addition, light and charm jets may produce fake $b$ tags. Apart from our signal $\bar{t} \bar{t} H H$ we therefore include as backgrounds processes where

(i) $Z$ and Higgs bosons decay into $b$-quark pairs, such as $t \bar{t} Z Z$ and $t \bar{t} h Z$;

(ii) one or both pairs of $b$ quarks are produced through gluon splittings in QCD, such as $t \bar{t} h b \bar{b}, t \bar{t} Z b \bar{b}$, or $t \bar{t} b \bar{b} b \bar{b}$

(iii) the leptonically decaying top quark is mimicked by a $W$ plus four $b$ jets, such as $W^{ \pm} b \bar{b} b \bar{b}+$ jets; and

\footnotetext{
${ }^{4}$ This statement is true provided we have a fixed order in mass dimension.

${ }^{5}$ The $68 \%$ constraints derived from Ref. [41] are $\kappa_{g g h h} \in$ $[-1.73,4.97] \times 10^{-8} \mathrm{GeV}^{-2}$, for an integrated luminosity of $3 / \mathrm{ab}$, while considering $d_{3}=1$ and $\kappa_{g}=0$, the SM values.
}

TABLE II. Renormalization and factorization scales used for the various processes, where $H_{T}$ is the scalar sum of the $p_{T}$ of the final state particles.

\begin{tabular}{lcc}
\hline \hline Process category & $\mu_{F}^{2}$ & $\mu_{R}^{2}$ \\
\hline$t \bar{t} H H, t \bar{t} Z Z, t \bar{t} H Z$ & $\frac{1}{4} H_{T}^{2}+2 m_{t}^{2}+\left\{2 m_{H}^{2}, 2 m_{Z}^{2}\right.$, & $\frac{1}{4} H_{T}^{2}+2 m_{t}^{2}$ \\
& $\left.m_{H}^{2}+m_{Z}^{2}\right\}$ & \\
$\begin{array}{c}t \bar{t} H b \bar{b}, t \bar{t} Z b \bar{b} \\
t \bar{t}+b \text { 's, } c \text { 's, } \\
\text { or light jets }\end{array}$ & $\frac{1}{4} H_{T}^{2}+m_{H, Z}^{2}+2 m_{t}^{2}$ & $\frac{1}{4} H_{T}^{2}+2 m_{t}^{2}$ \\
$\begin{array}{c}W+b \text { 's, } c \text { 's, } \\
\text { or light jets }\end{array}$ & $\frac{1}{4} H_{T}^{2}+2 m_{t}^{2}$ & $\frac{1}{4} H_{T}^{2}+2 m_{t}^{2}$ \\
\hline \hline
\end{tabular}

(iv) subdominant or fake backgrounds which can contribute to the total background yield, for example, $t \bar{t} c \bar{c} c \bar{c}$ and $W^{ \pm} c \bar{c} c \bar{c}$, misidentifying charm as $b$ quarks, or $t \bar{t} b \bar{b}, t \bar{t} h, t \bar{t} Z$, and $W^{ \pm} b \bar{b}$, all associated by light jets.

Because of their complexity and their large final-state multiplicity we chose to simulate signal and backgrounds with leading order matrix elements and consistently combine them with subsequent parton showers, to capture the

TABLE III. The generation level cross sections for the signal and background processes. We require the Higgs bosons to decay to a pair of $b / c$ quarks and the $Z$ bosons to all quarks. Furthermore, we require the $W^{ \pm}$bosons to decay leptonically. These branching ratios are included in these cross sections. For the signals, $\kappa_{\lambda}$ is the ratio of the Higgs self-coupling to the SM value and $\kappa_{\bar{t} t h h}$ is the coupling of the four point $t \bar{t} h h$ interaction. The processes with $b / c$ quarks in the final state in the matrix element level have a further requirement of $m_{b b / c c / b c}>50 \mathrm{GeV}$, $p_{T}(b / c)>25 \mathrm{GeV}, D$-parameter $>0.4,|y|<4.0$.

\begin{tabular}{lc}
\hline Channel & Cross section [pb] \\
\hline$t \bar{t} h h\left(\kappa_{\lambda}=1\right)$ & 0.015 \\
$t \bar{t} h h\left(\kappa_{\lambda}=2\right)$ & 0.020 \\
$t \bar{t} h h\left(\kappa_{\lambda}=0\right)$ & 0.012 \\
$t \bar{t} h h\left(\kappa_{\lambda}=-1\right)$ & 0.012 \\
$t \bar{t} h h\left(\kappa_{\lambda}=-2\right)$ & 0.014 \\
$t \bar{t} h h\left(\kappa_{t \bar{t} h h}=-0.003 \mathrm{GeV}^{-1}\right)$ & 0.175 \\
$t \bar{t} h h\left(\kappa_{t \bar{t} h}=0.003 \mathrm{GeV}^{-1}\right)$ & 0.132 \\
$\bar{t} \bar{t} b \bar{b} b \bar{b}$ & 0.174 \\
$t \bar{t} c \bar{c} c \bar{c}$ & 0.174 \\
$t \bar{t} b \bar{b}+$ jets & 46.30 \\
$t \bar{t} h b \bar{b}$ & 0.076 \\
$t \bar{t} h+$ jets & 12.825 \\
$t \bar{t} h Z$ & 0.045 \\
$t \bar{t} Z Z$ & 0.057 \\
$t \bar{t} Z b \bar{b}$ & 0.165 \\
$t \bar{t} Z+$ jets & 25.663 \\
$W^{ \pm} b \bar{b} b \bar{b}+$ jet & 0.036 \\
$W^{ \pm} c \bar{c} c \bar{c}+$ jet & 0.092 \\
\hline \hline
\end{tabular}


effect of QCD radiation and, in particular, of gluon splittings into heavy quarks.

We use SHERPA v2.2.5 [56,57] with the COMIX matrix element generator [58] and the parton shower based on Catani-Seymour splitting kernels [59,60]. The central CT14NLO parton distribution function (PDF) set [61] is used throughout. All jets contributing to the process classification, including $b$ and $c$ jets, are defined with the anti- $k_{T}$ algorithm [62] with

$$
p_{T}(j)>25 \mathrm{GeV}, \quad\left|y_{j}\right|<4.0 .
$$

We also require a minimal invariant mass $m_{b b / b c / c c} \geq$ $50 \mathrm{GeV}$ for all possible $b$ and $c$ pairs. Where necessary, we add matrix elements for final state with more jets through multijet merging according to [63-65], and a merging cut of $Q_{\text {cut }}=40 \mathrm{GeV}$. For the various processes we use the renormalization and factorization scales listed in Table II, where for merged samples we cluster back to the relevant core process before determining the scales.

Details of the generation cross sections for all signal and background samples are listed in Table III.

\section{ANALYSIS}

In Ref. [31], the $t \bar{t} h h$ channel was studied in the context of the $14 \mathrm{TeV}$ high-luminosity run of the LHC. In the
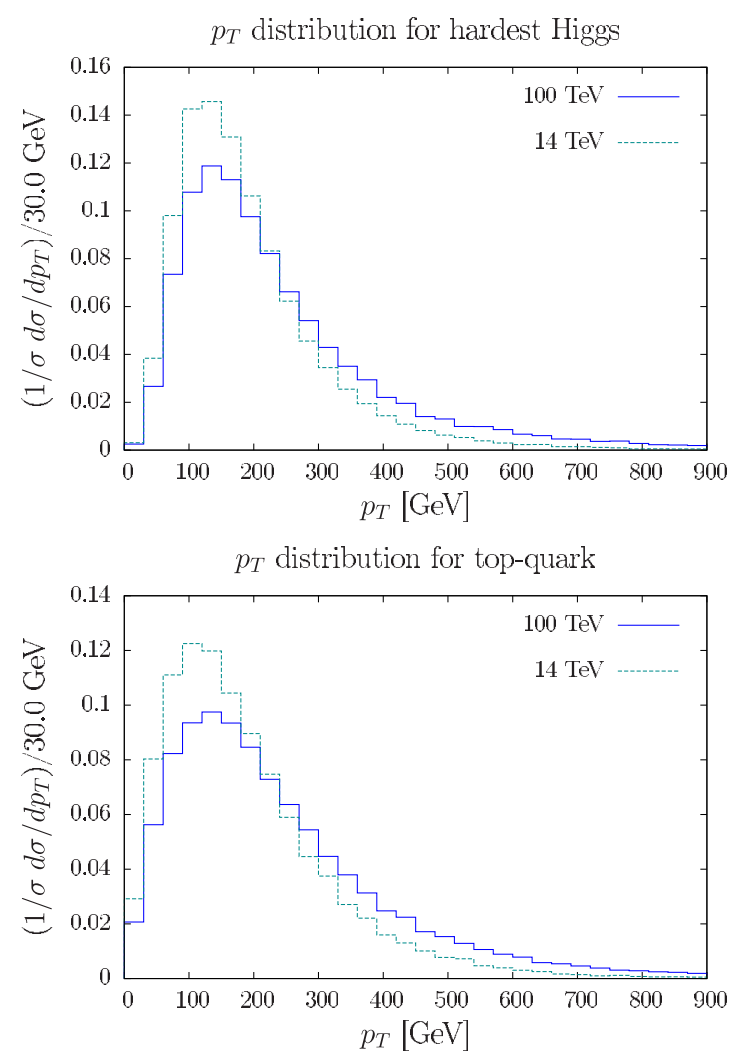

present work we revisit this analysis by focusing on the prospects of observing this channel at a possible future FCC-hh $100 \mathrm{TeV} p p$ collider. While our analysis strategy here is reminiscent of Ref. [31], we study the backgrounds in significantly greater detail.

At leading order, the $p p \rightarrow t \bar{t} h h$ cross section increases by a factor of $\sim 75$ for the SM scenario upon going from $14 \mathrm{TeV}$ to $100 \mathrm{TeV}$. However, from Fig. 3, we can see that the large increment in the total cross section does not translate into a significantly enhanced distribution for large transverse momenta. Hence, any advantage in the analysis is due to the increased total rate and to an improved reconstruction efficiency for highly energetic final-state objects.

To reconstruct the final state, jets are clustered with the anti- $k_{t}$ algorithm [62] in the FASTJET framework [66] and parameters

$$
R=0.4, \quad p_{T, j}>30 \mathrm{GeV}, \quad \text { and } \quad\left|\eta_{j}\right|<4.5 .
$$

We require our events to have at least eight jets, out of which exactly six must be $b$ tagged. For the $b$-tagged jets we demand that the distance between $B$-hadron and jet center fulfills

$$
\Delta R_{j, B}<0.2
$$

and that
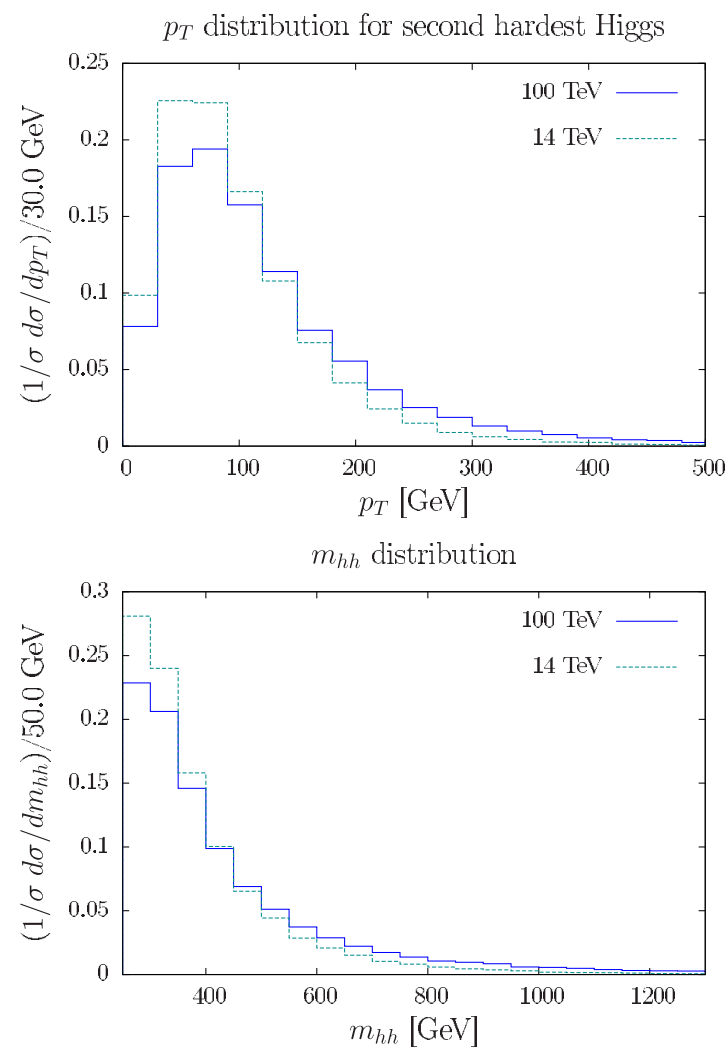

FIG. 3. Normalized distributions showing the $p_{T}$ spectra of the hardest and second hardest Higgs bosons and the top quark, and the invariant mass spectrum of the di-Higgs system, at the $14 \mathrm{TeV}$ and $100 \mathrm{TeV}$ colliders. 

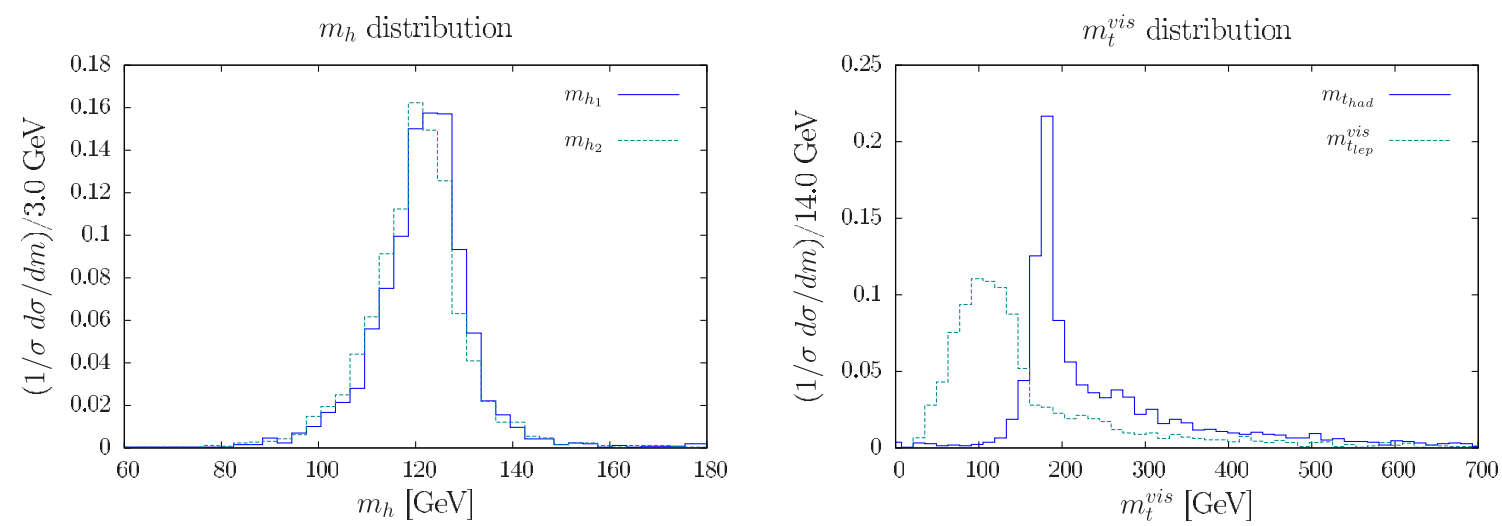

FIG. 4. Mass reconstruction of the hardest and the second-hardest Higgs bosons (left) and the hadronic and visible part of the leptonic top quarks (right).

$$
\left|y_{b}\right|<2.5 \text {. }
$$

For the $100 \mathrm{TeV}$ collider study, we assume a $b$-tagging efficiency of $80 \%$. To estimate the effect of fake $b$ tags, we assume a mistagging probability of $10 \%$ for $c$ jets and of $1 \%$ for light jets. We require exactly one lepton in each event with

$$
p_{T}(\ell)>10 \mathrm{GeV} \text { and }\left|y_{\ell}\right|<2.5 .
$$

To isolate the leptons we demand that the total hadronic activity around a cone of $\Delta R=0.3$ to be less than $10 \%$ of its $p_{T}$.

From Table III, it is clear that some of the backgrounds are much larger in cross sections than others. We note that the $t \bar{t} h+$ jets process already contains $t \bar{t} h b \bar{b}$. The same is true for $t \bar{t} Z+$ jets and $t \bar{t} Z b \bar{b}$. Thus, in order to avoid double counting, we focus on the $t \bar{t} h+$ jets and $t \bar{t}+$ jets channels. For the $t \bar{t} h / Z+$ jets, we consider the merged sample, where additional jets stem from QCD radiation, including the gluon splitting into $b$-quark pairs. To ensure that none of the additional jets contains more than one $B$ meson, we implement a further criterion, namely that the $B$ hadron closest to the jet axis satisfies

$$
x_{B}=\frac{\left|\vec{p}_{B}\right|}{\left|\vec{p}_{j}\right|} \times \frac{\vec{p}_{B} \cdot \vec{p}_{j}}{\left|\vec{p}_{B}\right|\left|\vec{p}_{j}\right|}>0.7 .
$$

This condition reflects the $b$-quark fragmentation, which is characterized by relatively low energy or momentum losses due to QCD radiation and, correspondingly, the fact that $B$ hadrons in a jet stemming from a $b$ quark carry the dominant fraction of the jet momentum. Obviously, this is not true for those jets, where a gluon splits into two $b$ quarks, which typically have relatively symmetric momentum fractions. Consequently, this criterion effectively suppresses "doubly tagged" $b$ jets, which contain two $b$ hadrons. The $b$-tagged jets failing to fulfill this criterion are considered as light jets, and we call those jets that fulfill it "good" $b$ jets. We therefore require events with exactly six good $b$-tagged jets, and all pairs of $b$-tagged jets must have an invariant mass greater than $50 \mathrm{GeV}$. We confirmed the results of some previous investigations using such a cut, for example in [67], which found that it suppresses each doubly tagged $b$ jet by more than about $80 \%$. As a consequence, we could confirm that these additional conditions on good $b$ jets render the effect of gluon jets tagged as $b$ jets due to gluon splitting negligibly small.

To ascertain that the events are ensuing from a $t \bar{t} h h$ topology, reconstructing most of the electroweak resonances is of essence. We follow Ref. [31] and define our two Higgs boson candidates by minimizing

$$
\chi_{H H}^{2}=\frac{\left(m_{b_{i}, b_{j}}-m_{h}\right)^{2}}{\Delta_{h}^{2}}+\frac{\left(m_{b_{k}, b_{l}}-m_{h}\right)^{2}}{\Delta_{h}^{2}},
$$

where $i \neq j \neq k \neq l$ run over all the six $b$-tagged jets. As parameters for this minimization we use $m_{h}=120 \mathrm{GeV}$ and $\Delta_{h}=20 \mathrm{GeV}$. The strange choice for $m_{h}$ warrants an elucidation. Because the Higgs bosons decay to $b$ quarks that essentially hadronize to $B$ mesons, the invisible decays of the latter shifts the reconstruction of the Higgs peak to smaller values. A different value of $m_{h}$ can be chosen after correcting explicitly for jet energy effects in $b$ jets. After minimizing $\chi_{H H}^{2}$, we require $\left|m_{b_{i}, b_{j}}-m_{h}\right|<\Delta_{h}$ and $\left|m_{b_{k}, b_{l}}-m_{h}\right|<\Delta_{h}$. The reconstructions of the hardest and the second hardest Higgs bosons are shown in Fig. 4 (left).

After finding the two Higgs bosons, we are left with two $b$-tagged jets. Because of the uncertainty in the longitudinal momentum $p_{z}$ of the neutrino, we only reconstruct the hadronic top, $t_{h}$. We consider the two remaining $b$-tagged jets and all other light jets to minimize the following $\chi^{2}$ :

$$
\chi_{t_{h}}^{2}=\frac{\left(m_{b_{i}, j_{k}, j_{l}}-m_{t}\right)^{2}}{\Delta_{t}^{2}},
$$

where $i$ runs over the remaining two $b$-tagged jets and $k \neq l$ denote the indices of all the light jets. For this minimization, we take $m_{t}=170 \mathrm{GeV}$ and $\Delta_{t}=40 \mathrm{GeV}$. We allow for a larger uncertainty as we demand the 
TABLE IV. Cut-flow table showing the cut efficiencies for the various cuts used for three signal scenarios and for the four dominant backgrounds.

\begin{tabular}{|c|c|c|c|c|c|c|c|}
\hline Cuts & $\kappa_{\lambda}=1$ & $\kappa_{\lambda}=2$ & $\kappa_{t \bar{t} h h}=-0.003$ & $t \bar{t} b \bar{b} b \bar{b}$ & $t \bar{t} h b \bar{b}$ & $\bar{t} \bar{t} Z h$ & $t \bar{t} Z b \bar{b}$ \\
\hline Trigger + isolation & 0.24 & 0.24 & 0.23 & 0.25 & 0.26 & 0.25 & 0.25 \\
\hline$>7$ jets & 0.56 & 0.57 & 0.67 & 0.68 & 0.61 & 0.55 & 0.60 \\
\hline $6 \operatorname{good} b$ jets & 0.019 & 0.020 & 0.018 & 0.009 & 0.014 & 0.004 & 0.003 \\
\hline $100 \mathrm{GeV}<m_{h_{1 / 2}}<140 \mathrm{GeV}$ & 0.88 & 0.89 & 0.84 & 0.38 & 0.58 & 0.68 & 0.37 \\
\hline $130 \mathrm{GeV}<m_{t_{h}}<210 \mathrm{GeV}$ & 0.30 & 0.28 & 0.38 & 0.30 & 0.27 & 0.28 & 0.23 \\
\hline$m_{t_{\text {lep }}^{\text {vis }}}<170 \mathrm{GeV}$ & 0.76 & 0.77 & 0.73 & 0.57 & 0.60 & 0.68 & 0.47 \\
\hline
\end{tabular}

hadronic top to be reconstructed from three jets. After minimizing $\chi_{t_{h}}^{2}$, we require $\left|m_{b_{i}, j_{k}, j_{l}}-m_{t}\right|<\Delta_{t}$. Finally, instead of fully reconstructing the leptonic top, we reconstruct the invariant mass of the last $b$ jet that is neither part of the two Higgs reconstructions nor of the hadronic top and the single isolated lepton. We further require the visible invariant mass to satisfy $m_{t_{\text {lep }}^{\text {vis }}}<m_{t}$. The reconstruction of the hadronic and the visible part of the leptonic top masses (before imposing the cuts) are shown in Fig. 4 (right). These two reconstructions ensure the complete obliteration of the $W^{ \pm}+$jets backgrounds. In Table IV, we show the effects of the various cuts for three signal scenarios $\left(\kappa_{\lambda}=1\right.$, 2 and $\left.\kappa_{\bar{t} \bar{h} h}=-0.003 \mathrm{GeV}^{-1}\right)$ and the four dominant backgrounds, i.e., $t \bar{t} b \bar{b} b \bar{b}, t \bar{t} h b \bar{b}, t \bar{t} Z h$, and $t \bar{t} Z b \bar{b}$.

Finally after imposing all cuts, we are left with the cross sections listed in Table V. For the case of $\lambda_{\mathrm{SM}}$, we obtain a signal over background ratio of $S / B \sim 0.14$ at leading order. For the design luminosity of 30/ab, this translates into a statistical significance, $S / \sqrt{B} \sim 6.3$ upon assuming

TABLE V. Cross sections (in $\mathrm{fb}$ ) for the various signal scenarios and backgrounds after all the cuts and requirements detailed in Table IV.

\begin{tabular}{lc}
\hline \hline Channel & Cross section [fb] \\
\hline$t \bar{t} h h\left(\kappa_{\lambda}=1\right)$ & 0.0091 \\
$t \bar{t} h h\left(\kappa_{\lambda}=2\right)$ & 0.0118 \\
$t \bar{t} h h\left(\kappa_{\lambda}=0\right)$ & 0.0071 \\
$t \bar{t} h h\left(\kappa_{\lambda}=-1\right)$ & 0.0072 \\
$t \bar{t} h h\left(\kappa_{\lambda}=-2\right)$ & 0.0083 \\
$t \bar{t} h h\left(\kappa_{\bar{t} \bar{t} h}=-0.003 \mathrm{GeV}^{-1}\right)$ & 0.1135 \\
$t \bar{t} h h\left(\kappa_{\bar{t}} h h=0.003 \mathrm{GeV}^{-1}\right)$ & 0.0912 \\
$t \bar{t} b \bar{b} b \bar{b}$ & 0.0217 \\
$t \bar{t} c \bar{c} c \bar{c}$ & $\lesssim \mathcal{O}\left(10^{-4}\right)$ \\
$t \bar{t} h+$ jets & 0.0333 \\
$t \bar{t} h Z$ & 0.0031 \\
$t \bar{t} Z Z$ & $\lesssim \mathcal{O}\left(10^{-4}\right)$ \\
$t \bar{t} Z+$ jets & 0.0035 \\
$W^{ \pm} b \bar{b} b \bar{b}+$ jet & $\lesssim \mathcal{O}\left(10^{-4}\right)$ \\
$W^{ \pm} c \bar{c} c \bar{c}+$ jet & $\lesssim \mathcal{O}\left(10^{-4}\right)$ \\
$W^{ \pm} b \bar{b}+$ jets & $\lesssim \mathcal{O}\left(10^{-4}\right)$ \\
Total background & 0.0623 \\
\hline \hline
\end{tabular}

no systematic uncertainties. Finally we feed these results into a log-likelihood CLs hypothesis test assuming the SM as the null hypothesis and also assuming no systematic uncertainties. At 68\% (95\%) confidence level, we find

$$
\begin{gathered}
-3.09<\kappa_{\lambda}<2.44\left(-3.60<\kappa_{\lambda}<3.16\right) \quad 3 / \mathrm{ab}, \\
-2.56<\kappa_{\lambda}<1.64\left(-2.83<\kappa_{\lambda}<2.06\right) \quad 30 / \mathrm{ab} .
\end{gathered}
$$

Assuming a flat 5\% (10\%) systematic uncertainty, the $68 \%$ confidence level limits change to

$$
\begin{gathered}
-3.20<\kappa_{\lambda}<2.60\left(-3.43<\kappa_{\lambda}<2.92\right) \quad 3 / \mathrm{ab}, \\
-2.89<\kappa_{\lambda}<2.15\left(-3.27<\kappa_{\lambda}<2.70\right) \quad 30 / \mathrm{ab} .
\end{gathered}
$$

Last, we also perform the same test on the $t \bar{t} h h$ four point vertex. Upon resorting to a model independent bound on the coupling, we obtain the following bounds at $68 \%$ (95\%) confidence level ${ }^{6}$ :

$$
\begin{aligned}
&- 0.53 \mathrm{TeV}^{-1}<\kappa_{i \bar{t} h h}<0.89 \mathrm{TeV}^{-1}\left(-0.81 \mathrm{TeV}^{-1}<\kappa_{\text {ẗthh }}\right. \\
&\left.<1.17 \mathrm{TeV}^{-1}\right) \quad 3 / \mathrm{ab}, \\
&- 0.25 \mathrm{TeV}^{-1}<\kappa_{\bar{t} \bar{t} h h}<0.61 \mathrm{TeV}^{-1}\left(-0.39 \mathrm{TeV}^{-1}\right. \\
&\left.<\kappa_{\bar{t} \bar{h} h}<0.75 \mathrm{TeV}^{-1}\right) \quad 30 / \mathrm{ab} .
\end{aligned}
$$

Upon considering a $5 \%(10 \%)$ systematic uncertainty, the $68 \%$ confidence level limits become

$$
\begin{aligned}
- & 0.59 \mathrm{TeV}^{-1}<\kappa_{\bar{t} \bar{t} h}<0.95 \mathrm{TeV}^{-1}\left(-0.71 \mathrm{TeV}^{-1}<\kappa_{\bar{t} \bar{h} h}\right. \\
& \left.<1.07 \mathrm{TeV}^{-1}\right) \quad 3 / \mathrm{ab}, \\
& -0.43 \mathrm{TeV}^{-1}<\kappa_{\bar{t} \bar{h} h}<0.78 \mathrm{TeV}^{-1}\left(-0.63 \mathrm{TeV}^{-1}\right. \\
& \left.<\kappa_{\bar{t} \bar{t} h}<0.99 \mathrm{TeV}^{-1}\right) \quad 30 / \mathrm{ab} .
\end{aligned}
$$

To see if we can gain additional sensitivity, we finally perform a multivariate analysis (MVA) with boosted

\footnotetext{
${ }^{6}$ Upon considering an inclusive analysis, Ref. [41] obtains $\kappa_{\bar{t} \bar{t} h} \in[-1.72,1.15] \mathrm{TeV}^{-1}$ at $68 \%$ C.L. at 3/ab. Whereas, upon considering the $m_{h h}$ variable, they obtain stronger bounds, $\left|\kappa_{t \bar{t} h h}\right|<0.14 \mathrm{Tev}^{-1}$ at $68 \%$ C.L. for the same integrated luminosity. Further optimization is thus possible for the $\bar{t} \bar{t} h h$ channel.
} 
decision trees (BDT) in the TMVA framework [68] with the following variables: reconstructed masses of both the Higgs bosons, $h_{1}, h_{2}$, reconstructed mass of the hadronic top quark, visible part of the reconstructed leptonic top quark, transverse momenta of these four objects, transverse momenta of the six $b$-tagged jets, hardest two light jets and the isolated lepton, the missing transverse energy and $\Delta R\left(h_{1} h_{2} / h_{i} t_{h} / h_{i} t_{\text {lep }}^{\mathrm{vis}}\right)$, where $i=1,2$, and the total number of jets. We train the SM $t \bar{t} h h$ sample with the dominant backgrounds, viz. $t \bar{t} b \bar{b} b \bar{b}, t \bar{t} h b \bar{b}, t \bar{t} h Z, t \bar{t} Z b \bar{b}$, and $t \bar{t} Z Z$. However, owing to a strong drop in efficiency due to several reconstructions and requirements, we are left with an inadequate number of Monte Carlo (MC) events for a proper training [69]. ${ }^{7}$ However, the various variables involved are mostly indiscernible and a BDT is not efficient in improving the sensitivity. For completeness, we find that our $S / B$ improves to $\sim 0.17$ with the statistical significance increasing to $\sim 6.4$. Thus, we did not pursue the MVA analysis further.

The results obtained above are assuming a nonlinear realization of the EFT. Furthermore, we do not marginalize over the other parameters while quoting the constraints. This may change our results to some degree. This first study is important to show the sensitivity of the complicated $t \bar{t} h h$ channel in the $6 b, 1 \ell+$ jets final state. It is worth mentioning that even though the constraint on the Higgs self-coupling is weaker than what obtains through the $p p \rightarrow h h$ production, the constraint on $\kappa_{\bar{t} t h h}$ is of the same order as the one obtained from the $p p \rightarrow h h$ channel as shown in Ref. [41]. This motivates us to combine multiple double Higgs production modes to constrain these couplings even better. Also from Ref. [70], where the couplings are varied one at a time, the $p p \rightarrow h h$ channel yields a constraint on $\kappa_{t \bar{t} h h}$ that is at least an order of magnitude weaker than the limit derived here. All these results encourage us to study the $t \bar{t} h h$ channel in other final states at the $100 \mathrm{TeV}$ collider and combine the results with the $p p \rightarrow h h$ analyses.

Before concluding, we want to comment on the perturbative order of our calculations. Reference [33] evaluated the impact of next-to-leading-order (NLO) $K$ factors on the total cross sections for the various double Higgs production processes, which are typically of the order of $25 \%$ for our channel. While for the signal processes, the NLO corrections are known and calculable with standard tools, this is not true for the significantly more involved background processes, i.e., $t \bar{t} b \bar{b} b \bar{b}(\mathrm{QCD}), t \bar{t} h(Z) b \bar{b}, t \bar{t} h(Z)+$ jets, etc. Because we want to treat all processes on identical footing,

\footnotetext{
${ }^{7}$ For all the channels except for $t \bar{t} h / Z+$ jets, we started with a million MC events. For the latter two we started with $10 \times 10^{6}$ $\left(22 \times 10^{6}\right)$ events for $t \bar{t} h(Z)+$ jets. For all the signal samples, we end up with $\sim 600-700 \mathrm{MC}$ events after all the cuts. For the various backgrounds, however, we end up with 20-200 MC events. These are not enough to properly train an MVA.
}

we did not include NLO corrections to the signal subset only. In addition, it is well known that multijet merging when applied correctly is very well capable of recovering the impact of higher-order corrections on shape observables. This is supported, e.g., by Figs. 4 and 5 in Ref. [33], where the bin-by-bin $K$ factors for the $p_{T}$ of the hardest (second-hardest) Higgs boson are constant within $10 \%$ or better at around $0.8(0.75)$ for $p_{T}$ values up to $350 \mathrm{GeV}$ $(300 \mathrm{GeV})$. Larger fluctuations in the tails can be traced back to even higher orders, in this case to the emission of more than one additional parton. Our study does not focus particularly on the tails of the transverse momentum distributions, and because our discriminatory observables (as listed in Table IV) are mostly invariant masses, we do not expect any significant changes in shapes due to NLO effects. To account for NLO effects on total cross sections, we add an additional $30 \%$ systematic uncertainty on the total rates, and we find at $68 \%$ C.L. and at $30 \mathrm{ab}^{-1}$

$$
-4.23<\kappa_{\lambda}<3.98, \quad-1.18 \mathrm{TeV}^{-1}<\kappa_{t \bar{t} h h}<1.54 \mathrm{TeV}^{-1} .
$$

\section{SUMMARY AND CONCLUSIONS}

One of the most important tasks after the discovery of the Higgs boson and after studying its couplings with gauge bosons and third-generation fermions is to understand the interactions of the scalar sector underlying electroweak symmetry breaking in more detail. One of its cornerstones is the trilinear interaction of the Higgs boson $\kappa_{\lambda}$. Within most realistic extensions of the Standard Model one does not expect a modified Higgs self-interaction in isolation, but modifications of various couplings, i.e., the presence of many additional operators. Such new operators would simultaneously contribute to di-Higgs production processes, e.g., $p p \rightarrow h h$, and would therefore result in blind directions in a global fit. To overconstrain the system of operators expected in extensions of the Standard Model it is consequently of crucial importance to measure as many multi-Higgs final states as possible.

We have revisited the sensitivity of the process $p p \rightarrow$ $t \bar{t} h h$ at a future circular collider with $\sqrt{s}=100 \mathrm{TeV}$. To take into account deformations of the Standard Model, we varied the two operators $\kappa_{\lambda} \lambda_{\mathrm{SM}} h^{3}$ and $\kappa_{\bar{t} t h h}\left(\bar{t}_{L} t_{R} h^{2}+\right.$ H.c. $)$ independently. While the signal cross section increases by a factor of 75 between $\sqrt{s}=14 \mathrm{TeV}$ and $\sqrt{s}=100 \mathrm{TeV}$, the total background before cuts increases by $\sim 40$. Each background has a different enhancement factor, and this total factor becomes $\sim 80$ if we do not take into account the $W^{ \pm}+$jets backgrounds that are completely negligible after the analysis. Hence, we surveyed a comprehensive list of backgrounds and found that the two operators can be constrained to $-2.14<\kappa_{\lambda}<1.60$ and $-0.25 \mathrm{TeV}^{-1}<$ $\kappa_{\bar{t} \bar{t} h}<0.61 \mathrm{TeV}^{-1}$ for an integrated luminosity of $30 / \mathrm{ab}$ at $68 \%$ C.L. assuming zero systematics. 
While the limit on $k_{\lambda}$ is not competitive to the predicted limits from the processes $p p \rightarrow h h[41,43,71,72]$ or $p p \rightarrow$ $h h j$ [73] for a $100 \mathrm{TeV}$ collider, the fact that both coefficients $\kappa_{\lambda}$ and $\kappa_{\bar{t} t h h}$ are contributing at tree level to $\bar{t} \bar{t} h h$ production means that this process is of significant importance to include in an agnostic global fit for nonlinear EFT parameters along with the parameters affecting the $\bar{t} \bar{t}, g g h$, and $g g h h$ vertices. However, it is important to realize that these vertices can already be constrained from the gluon-fusion and vector boson fusion (VBF) productions ( $p p \rightarrow h h, h h j$, $h h j j$ ) and the $t \bar{t} h h$ process will help in disentangling these further owing to having different linear combinations in the couplings.

\section{ACKNOWLEDGMENTS}

We thank Silvan Kuttimalai for help with Sherpa. We also thank Shilpi Jain, Jonas Lindert, and Marek Schoenherr for helpful discussions during various stages of this work. S. B. is supported by a Durham Junior Research Fellowship CO-FUNDed by Durham University and the European Union, under Grant No. 609412.
[1] G. Aad et al. (ATLAS Collaboration), Phys. Lett. B 716, 1 (2012).

[2] S. Chatrchyan et al. (CMS Collaboration), Phys. Lett. B 716, 30 (2012).

[3] G. Aad et al. (ATLAS Collaboration), Eur. Phys. J. C 75, 476 (2015); 76, 152(E) (2016).

[4] G. Aad et al. (ATLAS Collaboration), Eur. Phys. J. C 76, 658 (2016).

[5] A. M. Sirunyan et al. (CMS Collaboration), Phys. Lett. B 775, 1 (2017).

[6] V. Khachatryan et al. (CMS Collaboration), Phys. Lett. B 759, 672 (2016).

[7] G. Aad et al. (ATLAS and CMS Collaborations), J. High Energy Phys. 08 (2016) 045.

[8] A. M. Sirunyan et al. (CMS Collaboration), Phys. Rev. Lett. 121, 121801 (2018).

[9] M. Aaboud et al. (ATLAS Collaboration), Phys. Lett. B 784, 173 (2018).

[10] C. Englert, R. Kogler, H. Schulz, and M. Spannowsky, Eur. Phys. J. C 76, 393 (2016).

[11] J. de Blas, M. Ciuchini, E. Franco, S. Mishima, M. Pierini, L. Reina, and L. Silvestrini, J. High Energy Phys. 12 (2016) 135 .

[12] A. Butter, O. J. P. Éboli, J. Gonzalez-Fraile, M. C. GonzalezGarcia, T. Plehn, and M. Rauch, J. High Energy Phys. 07 (2016) 152.

[13] J. Ellis, C. W. Murphy, V. Sanz, and T. You, J. High Energy Phys. 06 (2018) 146.

[14] J. J. van der Bij, Nucl. Phys. B267, 557 (1986).

[15] G. Degrassi, M. Fedele, and P. P. Giardino, J. High Energy Phys. 04 (2017) 155.

[16] G. D. Kribs, A. Maier, H. Rzehak, M. Spannowsky, and P. Waite, Phys. Rev. D 95, 093004 (2017).

[17] W. Bizon, M. Gorbahn, U. Haisch, and G. Zanderighi, J. High Energy Phys. 07 (2017) 083.

[18] G. Degrassi, P. P. Giardino, F. Maltoni, and D. Pagani, J. High Energy Phys. 12 (2016) 080.

[19] F. Maltoni, D. Pagani, A. Shivaji, and X. Zhao, Eur. Phys. J. C 77, 887 (2017).

[20] U. Baur, T. Plehn, and D. L. Rainwater, Phys. Rev. Lett. 89, 151801 (2002).
[21] U. Baur, T. Plehn, and D. L. Rainwater, Phys. Rev. D 67, 033003 (2003).

[22] M. J. Dolan, C. Englert, and M. Spannowsky, J. High Energy Phys. 10 (2012) 112.

[23] J. Baglio, A. Djouadi, R. Gröber, M. M. Mühlleitner, J. Quevillon, and M. Spira, J. High Energy Phys. 04 (2013) 151.

[24] Q.-H. Cao, G. Li, B. Yan, D.-M. Zhang, and H. Zhang, Phys. Rev. D 96, 095031 (2017).

[25] A. Adhikary, S. Banerjee, R. K. Barman, B. Bhattacherjee, and S. Niyogi, J. High Energy Phys. 07 (2018) 116.

[26] A. Abada et al., FCC, Vol. 3: The Hadron Collider (FCC-hh), Report No. CERN-ACC-2018-0058, 2019.

[27] CMS Collaboration, Prospects for $\mathrm{HH}$ measurements at the HL-LHC, Report No. CMS-PAS-FTR-18-019, 2018.

[28] M. J. Dolan, C. Englert, N. Greiner, and M. Spannowsky, Phys. Rev. Lett. 112, 101802 (2014).

[29] M. J. Dolan, C. Englert, N. Greiner, K. Nordstrom, and M. Spannowsky, Eur. Phys. J. C 75, 387 (2015).

[30] F. Bishara, R. Contino, and J. Rojo, Eur. Phys. J. C 77, 481 (2017).

[31] C. Englert, F. Krauss, M. Spannowsky, and J. Thompson, Phys. Lett. B 743, 93 (2015).

[32] T. Liu and H. Zhang, arXiv:1410.1855.

[33] R. Frederix, S. Frixione, V. Hirschi, F. Maltoni, O. Mattelaer, P. Torrielli, E. Vryonidou, and M. Zaro, Phys. Lett. B 732, 142 (2014).

[34] D. B. Kaplan, H. Georgi, and S. Dimopoulos, Phys. Lett. 136B, 187 (1984).

[35] G. Panico and A. Wulzer, Lect. Notes Phys. 913, 1 (2016).

[36] M. Chala, C. Krause, and G. Nardini, J. High Energy Phys. 07 (2018) 062.

[37] R. Grober and M. Muhlleitner, J. High Energy Phys. 06 (2011) 020.

[38] M. Gillioz, R. Grober, C. Grojean, M. Muhlleitner, and E. Salvioni, J. High Energy Phys. 10 (2012) 004.

[39] W. Yao, in Proceedings of the 2013 Community Summer Study on the Future of U.S. Particle Physics: Snowmass on the Mississippi (CSS2013): Minneapolis, MN, USA, 2013 (2013).

[40] A. J. Barr, M. J. Dolan, C. Englert, D. E. Ferreira de Lima, and M. Spannowsky, J. High Energy Phys. 02 (2015) 016. 
[41] A. Azatov, R. Contino, G. Panico, and M. Son, Phys. Rev. D 92, 035001 (2015).

[42] H.-J. He, J. Ren, and W. Yao, Phys. Rev. D 93, 015003 (2016).

[43] R. Contino et al., CERN Yellow Report No. CERN-TH2016-113, 2017, pp. 255-440.

[44] A. Abada et al., FCC, Vol. 1: Physics opportunities, CERN Report No. CERN-ACC-2018-0056, 2018.

[45] G. Buchalla, M. Capozi, A. Celis, G. Heinrich, and L. Scyboz, J. High Energy Phys. 09 (2018) 057.

[46] G. Aad et al. (ATLAS Collaboration), arXiv:1906.02025.

[47] F. Feruglio, Int. J. Mod. Phys. A 08, 4937 (1993).

[48] J. Bagger, V. D. Barger, K.-m. Cheung, J. F. Gunion, T. Han, G. A. Ladinsky, R. Rosenfeld, and C. P. Yuan, Phys. Rev. D 49, 1246 (1994).

[49] V. Koulovassilopoulos and R. S. Chivukula, Phys. Rev. D 50, 3218 (1994).

[50] G. Buchalla and O. Cata, J. High Energy Phys. 07 (2012) 101.

[51] G. Buchalla, O. Catà, and C. Krause, Nucl. Phys. B880, 552 (2014); 913, 475(E) (2016).

[52] G. F. Giudice, C. Grojean, A. Pomarol, and R. Rattazzi, J. High Energy Phys. 06 (2007) 045.

[53] D. Binosi, J. Collins, C. Kaufhold, and L. Theussl, Comput. Phys. Commun. 180, 1709 (2009).

[54] A. Alloul, B. Fuks, and V. Sanz, J. High Energy Phys. 04 (2014) 110.

[55] F. Goertz, A. Papaefstathiou, L. L. Yang, and J. Zurita, J. High Energy Phys. 04 (2015) 167.

[56] T. Gleisberg, S. Hoeche, F. Krauss, A. Schalicke, S. Schumann, and J.-C. Winter, J. High Energy Phys. 02 (2004) 056.

[57] T. Gleisberg, S. Hoeche, F. Krauss, M. Schonherr, S. Schumann, F. Siegert, and J. Winter, J. High Energy Phys. 02 (2009) 007.
[58] T. Gleisberg and S. Hoeche, J. High Energy Phys. 12 (2008) 039.

[59] Z. Nagy and D. E. Soper, J. High Energy Phys. 09 (2007) 114.

[60] S. Schumann and F. Krauss, J. High Energy Phys. 03 (2008) 038.

[61] S. Dulat, T.-J. Hou, J. Gao, M. Guzzi, J. Huston, P. Nadolsky, J. Pumplin, C. Schmidt, D. Stump, and C. P. Yuan, Phys. Rev. D 93, 033006 (2016).

[62] M. Cacciari, G. P. Salam, and G. Soyez, J. High Energy Phys. 04 (2008) 063.

[63] S. Catani, F. Krauss, R. Kuhn, and B. R. Webber, J. High Energy Phys. 11 (2001) 063.

[64] F. Krauss, J. High Energy Phys. 08 (2002) 015.

[65] S. Hoeche, F. Krauss, S. Schumann, and F. Siegert, J. High Energy Phys. 05 (2009) 053.

[66] M. Cacciari, G. P. Salam, and G. Soyez, Eur. Phys. J. C 72, 1896 (2012).

[67] D. Goncalves, F. Krauss, and R. Linten, Phys. Rev. D 93, 053013 (2016).

[68] A. Hoecker, P. Speckmayer, J. Stelzer, J. Therhaag, E. von Toerne, H. Voss, M. Backes, T. Carli, O. Cohen, A. Christov et al., arXiv:physics/0703039.

[69] D. Ciupke, 2012, http://www.desy.de/f/students/2012/reports/ david_ciupke.pdf.gz.

[70] A. Carvalho, M. Dall'Osso, P. De Castro Manzano, T. Dorigo, F. Goertz, M. Gouzevich, and M. Tosi, arXiv:1608 .06578 .

[71] A. Papaefstathiou, Phys. Rev. D 91, 113016 (2015).

[72] D. Gonçalves, T. Han, F. Kling, T. Plehn, and M. Takeuchi, Phys. Rev. D 97, 113004 (2018).

[73] S. Banerjee, C. Englert, M. L. Mangano, M. Selvaggi, and M. Spannowsky, Eur. Phys. J. C 78, 322 (2018). 